\title{
Electron-positron pair production in the external electromagnetic field of colliding relativistic heavy ions
}

\author{
A. Aste ${ }^{1}$, G. Baur ${ }^{2}$, K. Hencken ${ }^{1}$, D. Trautmann ${ }^{1}$ and G. Scharf ${ }^{3}$ a \\ 1 Institute for Theoretical Physics, University of Basel, Klingelbergstrasse 82, 4054 Basel, Switzerland \\ , 2 Institut für Kernphysik (Theorie), Forschungszentrum Jülich, 52425 Jülich, Germany \\ ${ }^{3}$ Institute for Theoretical Physics, University of Zürich, Winterthurerstrasse 190, 8057 Zürich, Switzerland
}

November 30, 2001

\begin{abstract}
The results concerning the $e^{+} e^{-}$production in peripheral highly relativistic heavy-ion collisions presented in a recent paper by Baltz et al. are rederived in a very straightforward manner. It is shown that the solution of the Dirac equation directly leads to the multiplicity, i.e. to the total number of electronpositron pairs produced by the electromagnetic field of the ions, whereas the calculation of the single pair production probability is much more involved. A critical observation concerns the unsolved problem of seemingly absent Coulomb corrections (Bethe-Maximon corrections) in pair production cross sections. It is shown that neither the inclusion of the vacuum-vacuum amplitude nor the correct interpretation of the solution of the Dirac equation concerning the pair multiplicity is able the explain (from a fundamental point of view) the absence of Coulomb corrections. Therefore the contradiction has to be accounted to the treatment of the high energy limit.

Keywords: Relativistic heavy-ion collisions; Electromagnetic pair production; Eikonal approximation; Coulomb corrections; S-matrix theory

PACS. Field theory 11.10.-z - Quantum electrodynamics 12.20.-m - S-matrix theory 11.55-m - Relativistic scattering theory 11.80.-m - Relativistic heavy-ion collisions 25.75.-q-Other topics in atomic and molecular collision processes and interactions $34.90 .+\mathrm{q}$
\end{abstract}

\section{Introduction}

The problem of $e^{+} e^{-}$pair production by the collision of highly relativistic nuclei has attracted a lot of interest during the past few years, since this process will have implications for future experiments performed at new facilities as BNL's RHIC and CERN's LHC. From the theoretical side, the result that the Dirac equation can be solved exactly in the electromagnetic background field created by two nuclei in the limit where the two nuclei are 'ultrarelativistic' [1, 2], seemed to lead to the unexpected consequence that the single pair cross section is equal to its Born value (given by the second order diagram in Fig. 2) 17. The term 'ultrarelativistic' is used here for the limiting case where the Lorentz factor $\gamma$ becomes large $(\gamma \rightarrow \infty)$, i. e. the velocity of the colliding ions approaches the speed of light. Of course the problem of pair production in an external field goes back to the beginning of QED [3, 4]. In connection with the relativistich heavy ion colliders it was found that the impact parameter dependent probability in per-

\footnotetext{
a Work supported by Swiss National Science Foundation.

1 Note that this is only true for the total cross section. As it was shown in [6], this is not true for impact parameter dependent probabilities.
}

turbation theory can become larger than one, which was shown to result in multiple pair production [5,6, 6, 8, 9 .

A series of papers on the same topic followed 110,11, 12, 13 with the aim to show that there must be an error in the interpretation of the results found in [1]. An interesting observation was finally made in a paper by Baltz et al. 14, where it was shown that the expression for pair production derived from the results in [1] describes the total number of produced pairs, and not the single pair production. Additionally, the importance of taking into account the vacuum-vacuum transition amplitude when going over from wave mechanics to the full external field problem with quantized electron field was pointed out, as was already discussed in [8,9]. A recent work which treats the structure of Coulomb and unitarity corrections to the single and multiple pair production is 15 .

We will derive in this paper the main results obtained in 14 in a very compact way using a different field theoretical point of view. The correct expression for the multiplicity can be derived indeed in a very straightforward manner from the fundamental equations which define the S-matrix for the external field problem. We establish the connection between the single-particle matrix elements from the solution of the Dirac equation and the pair pro- 
duction in a full many-body theory. Furthermore, we give a strong argument which highlights that the results provided from the theory of fermions in a classical electromagnetic background field are insufficient to explain the dicrepancies concerning Coulomb corrections in the literature.

It is therefore mandatory to understand the correct interpretation of the results obtained from 'infinite $\gamma$ ' calculations and their implications on real electromagnetic processes, as it was suggested in the work of Lee and Milstein [13 where they have shown that Coulomb corrections can be obtained by the correct regularization of matrix elements.

\section{The external field approximation for QED}

For highly relativistic heavy-ion collisions one usually assumes that the ions of electric charge $Z_{1} e$ and $Z_{2} e$ are sufficiently energetic and massive so that the deviation from straight-line trajectories can be neglected. The trajectories of the two ions are then, respectively, $z= \pm t(c=1)$, $\mathbf{x}_{\perp}=\mathbf{b} / 2$, and there is a gauge in which the potential of the two ions has the following form 16

$$
\begin{gathered}
A^{\mu}(t, \mathbf{x})=Z_{1} e \delta\left(v_{+} x\right) v_{+}^{\mu} \log \left(\frac{\left(\mathbf{x}_{\perp}-\mathbf{b} / 2\right)^{2}}{\mathbf{b}^{2}}\right) \\
+Z_{2} e \delta\left(v_{-} x\right) v_{-}^{\mu} \log \left(\frac{\left(\mathbf{x}_{\perp}+\mathbf{b} / 2\right)^{2}}{\mathbf{b}^{2}}\right)
\end{gathered}
$$

where $v_{ \pm}^{\mu}=(1,0,0, \pm 1) / \sqrt{2}$. The fact that the two electromagnetic fields are compressed due to the Lorentz contraction to a sheet in the $b$ plane, together with their structure in space-time allows for the exact solution of the Dirac equation in this case.

We will deal in the following with the general external field problem without referring to the special form of the electromagnetic field created by ultrarelativistic ions as given by Eq. (1), in order to clarify how the solution of the Dirac equation for the electron wave function and the full S-matrix for the quantized electron field in an external electromagnetic field are related to each other.

\subsection{Construction of the S-matrix}

In order to construct the full S-matrix of QED in the external field approximation, we start from a one-particle dynamics, defined by a time-dependent Hamiltonian acting on a state $\Psi$ described by a Dirac spinor $\Psi(t, \mathbf{x})$

$$
H(t)=H_{0}+V(t),
$$

where $V(t)$ contains the interaction with the external electromagnetic field given by

$$
V(t, \mathbf{x})=e(\phi(t, \mathbf{x})-\alpha \mathbf{A}(t, \mathbf{x})) .
$$

The potentials are assumed to vanish for $t \rightarrow \pm \infty$ in such a way that the wave operators exist as strong limits

$$
W_{\substack{\text { in } \\ \text { out }}}=\lim _{t \rightarrow \pm \infty} U(t, 0)^{+} e^{-i H_{0} t}
$$

and define a unitary S-matrix $S=W_{o u t}^{+} W_{i n}$. Since we presuppose free dynamics for $t \rightarrow \pm \infty$, we can base second quantization on the Fock representation of the free Dirac field. The one-particle sector of the Fock space then consists naturally of a positive spectral subspace (electrons) and negative spectral subspace (positrons) of the Hilbert space of Dirac four-spinors

$$
\mathcal{H}_{1}=\left(L^{2}\left(\mathcal{R}^{3}\right)\right)^{4}=P_{+}^{0} \mathcal{H}_{1} \oplus P_{-}^{0} \mathcal{H}_{1} .
$$

with the standard inner product. Here, $P_{ \pm}^{0}$ denote the corresponding spectral projection operators of the free Dirac Hamiltonian. The quantized Dirac field is then defined as usual in the Schrödinger picture

$$
\psi(f)=b\left(P_{+}^{0} f\right)+d\left(P_{-}^{0} f\right)^{+}=b\left(f_{+}\right)+d\left(f_{-}\right)^{+} .
$$

The electron annihilation operator $b\left(f_{+}\right)$destroys an electron with wave function $f_{+}$, and the positron emission operator $d\left(f_{-}\right)^{+}$produces a positron with wave function $f_{-}$. The annihilation and emission operators fulfil the fermionic anticommutation relations

$\left\{b\left(f_{+}\right), b\left(g_{+}\right)^{+}\right\}=\left(f_{+}, g_{+}\right), \quad\left\{d\left(f_{-}\right)^{+}, d\left(g_{-}\right)\right\}=\left(f_{-}, g_{-}\right)$,

and the Fock vacuum $\Omega$ is defined by

$$
b\left(f_{+}\right) \Omega=d\left(f_{-}\right) \Omega=0 \quad \forall f \in \mathcal{H}_{1} .
$$

Note that $b\left(f_{+}\right)$depends antilinearly on $f_{+}$, whereas $d\left(f_{-}\right)$ depends linearly on $f_{-}$. This explains the slight difference in the commutation relations for electron and positron operators in (7). We mention that naturally, the widely used field operators can be defined which correspond to the operators introduced above according to

$$
\begin{aligned}
& b\left(f_{+}\right)^{+}=\int d^{3} x b(\mathbf{x})^{+} f_{+}(\mathbf{x}), \\
& d\left(f_{-}\right)^{+}=\int d^{3} x d(\mathbf{x})^{+} f_{-}^{*}(\mathbf{x})
\end{aligned}
$$

or, given an arbitrary orthonormal basis $f_{j}$ in $P_{+}^{0} \mathcal{H}_{1}$ and $g_{k}$ in $P_{-}^{0} \mathcal{H}_{1}$,

$$
b^{+}(\mathbf{x})=\sum_{j} b\left(f_{j}\right)^{+} f_{j}^{*}(\mathbf{x}), \quad d^{+}(\mathbf{x})=\sum_{k} d\left(g_{k}\right)^{+} g_{k}(\mathbf{x})
$$

such that $\psi(\mathbf{x})=b(\mathbf{x})+d(\mathbf{x})^{+}$fulfils the well-known distributional identity

$$
\left\{\psi(\mathbf{x}), \psi\left(\mathbf{x}^{\prime}\right)^{+}\right\}=\mathbf{1}_{4} \delta^{(3)}\left(\mathbf{x}-\mathbf{x}^{\prime}\right) .
$$

The one-particle S-matrix, obtained via its perturbation series

$$
S=\sum_{n=0}^{\infty}(-i)^{n} \int_{-\infty}^{+\infty} d t_{1} \int_{-\infty}^{t_{1}} d t_{2} \ldots \int_{-\infty}^{t_{n-1}} d t_{n} \tilde{V}\left(t_{1}\right) \ldots \tilde{V}\left(t_{n}\right)
$$

where

$$
\tilde{V}(t)=e^{i H_{0} t} V(t) e^{-i H_{0} t},
$$


or by solving directly the Dirac equation with the external field, can be written as

$$
S=\left(\begin{array}{c}
S_{++} S_{+-} \\
S_{-+} S_{--}
\end{array}\right), \quad S^{-1}=S^{+}=\left(\begin{array}{c}
S_{++}^{+} S_{+-}^{+} \\
S_{-+}^{+}
\end{array}\right)
$$

if we define $S_{ \pm \pm}=P_{ \pm}^{0} S P_{ \pm}^{0}$ and $S_{ \pm \pm}^{+}=P_{ \pm}^{0} S^{+} P_{ \pm}^{0}$. It is often said that $S_{++}$and $S_{--}$are related to electron and positron scattering, whereas $S_{+-}$and $S_{-+}$are related to pair creation and annihilation. But the connection of $S_{ \pm \pm}$ to different measurable quantities has to be treated carefully. It should be stressed that the one-particle Dirac theory has no consistent physical interpretation, but only the corresponding second quantized many-particle theory in Fock space which we are now going to discuss.

If one lifts the one-particle Hamiltonian with indefinite energy spectrum to the Fock space

$$
\mathrm{H}=\sum_{j k}\left[\left(f_{j}, H f_{k}\right) b\left(f_{j}\right)^{+} b\left(f_{k}\right)-\left(g_{j}, H g_{k}\right) d\left(g_{k}\right)^{+} d\left(g_{j}\right)\right],
$$

then the Dirac field operator fulfils the Heisenberg equations of motion

$$
\begin{array}{r}
i \frac{d}{d t} \psi\left(U^{-1}(t, 0) f\right)=\left[\psi\left(U^{-1}(t, 0) f\right), \mathrm{H}\right], \\
i \frac{d}{d t} \psi\left(U^{-1}(t, 0) f\right)^{+}=\left[\psi\left(U^{-1}(t, 0) f\right)^{+}, \mathrm{H}\right] .
\end{array}
$$

Roughly speaking, (16) simply accounts for the fact that the total energy of a general Fock state should be given by the sum of energies of each single particle. Note also the important minus sign in 16 .

The definition (8) of the Fock vacuum would lead in the case of time-dependent external fields to Fock representations which continuously change in time. To avoid this somewhat odd construction, we retreat to scattering theory by defining the second quantized S-matrix S in correspondence to the Heisenberg equations of motion (18) by

$$
\begin{gathered}
\psi\left(S^{+} f\right)=\mathrm{S}^{-1} \psi(f) \mathrm{S} \\
\psi\left(S^{+} f\right)^{+}=\mathrm{S}^{-1} \psi(f)^{+\mathrm{S}} \quad \forall f \in \mathcal{H}_{1} .
\end{gathered}
$$

It is even possible to give an explicit normally ordered expression for $S$ 17, 18 .

$$
\begin{aligned}
\mathrm{S}=C & e^{S_{+-} S_{--}^{-1} b^{+} d^{+}}: e^{\left(S_{++}^{+-1}-1\right) b^{+} b}: \times \\
& : e^{\left(1-S_{--}^{-1}\right) d d^{+}}: e^{S_{--}^{-1} S_{-+} d b},
\end{aligned}
$$

where the terms in the exponents are a shorthand for the operators

$$
\begin{aligned}
& A b^{+} b \equiv \sum_{j k}\left(f_{j}, A f_{k}\right) b\left(f_{j}\right)^{+} b\left(f_{k}\right), \\
& A b^{+} d^{+} \equiv \sum_{j k}\left(f_{j}, A g_{k}\right) b\left(f_{j}\right)^{+} d\left(g_{k}\right)
\end{aligned}
$$

and similarly for $A d d^{+}$and $A d b$. Note that $S_{--}^{-1}$ is the inverse of the matrix $S_{--}$alone and therefore not identical to $S_{--}^{+}=P_{-}^{0} S^{-1} P_{-}^{0}$. $C$ denotes the vacuum-vacuum transition amplitude $(\Omega, \mathrm{S} \Omega)$. This vacuum-vacuum transition amplitude is always present, but whereas it has absolute value one, e.g., for static fields (and is therefore dropped in the calculations), $C$ is indispensable for the unitarity of $\mathrm{S}$, as has been verified in [14] by perturbation theory. In our case where two static Coulomb fields are moving relatively to each other, the lowest order contribution to the vacuum-vacuum transition amplitude is given by a loop graph shown in Fig.1. It is a well-known result that all vacuum graphs appearing in pair production amplitudes (see the example in Fig. 11) can be absorbed in the factor $C$. Additionally, the lowest order contribution is finite due to gauge invariance, whereas higher order contributions to the vacuum-vacuum transition amplitude are finite due to power counting arguments. Therefore, the theory is free of any regularization problems.
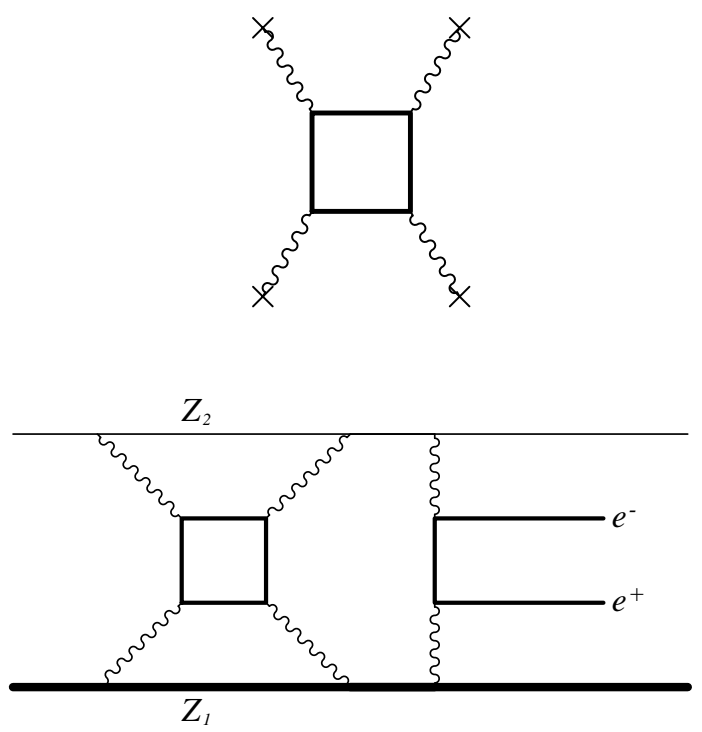

Fig. 1. Lowest order contribution to the vacuum-vacuum transition amplitude and single pair production graph containing a vacuum bubble.

Equation (21) is the important link showing the relation between the (single-particle) $S$ matrix from the solution of the Dirac equation and the general many-body theory.

We finally mention that the S-matrix $\mathrm{S}$ in Fock space exists, if and only if $S_{+-}$is a Hilbert-Schmidt operator. This condition is not satisfied with the potential (11) as it stands, but it can be achieved by appropriate regularization.

\subsection{Multiplicity}

From (19) it follows immediately

$$
b(f) \mathrm{S}=\mathrm{S}\left[b\left(S_{++}^{+} f\right)+d\left(S_{-+}^{+} f\right)^{+}\right],
$$


a simple identity which allows to derive easily the average number of electron-positron pairs produced by the external field. The number of pairs in the final state is equal to the number of electrons in the final state, which can be calculated from the electron (or positron) number operator

$$
N_{e}=\sum_{n} b\left(f_{n}\right)^{+} b\left(f_{n}\right), \quad N_{p}=\sum_{n} d\left(g_{n}\right)^{+} d\left(g_{n}\right) .
$$

Therefore, we obtain for the number of pairs in the final state created by the external field out of the vacuum $\mathrm{S} \Omega$

$$
\begin{aligned}
N & =\left(\mathrm{S} \Omega, \sum_{n} b\left(f_{n}\right)^{+} b\left(f_{n}\right) \mathrm{S} \Omega\right) \\
& =\sum_{n}\left(b\left(f_{n}\right) \mathrm{S} \Omega, b\left(f_{n}\right) \mathrm{S} \Omega\right) .
\end{aligned}
$$

Using (24), this can be written as

$$
\begin{aligned}
N=\sum_{n}\left(d\left(S_{-+}^{+} f_{n}\right) \Omega\right. & \left., d\left(S_{-+}^{+} f_{n}\right) \Omega\right)=\sum_{n}\left(f_{n}, S_{+-} S_{-+}^{+} f_{n}\right) \\
= & \operatorname{Tr}\left(S_{+-} S_{-+}^{+}\right)
\end{aligned}
$$

or equivalently, using the positron number operator

$$
N=\operatorname{Tr}\left(S_{-+} S_{+-}^{+}\right) .
$$

Therefore, the results obtained from the solution of the Dirac equation for $S_{-+}$傮 must not be misinterpreted as the single pair production amplitude. Furthermore, this result for which a lengthy derivation has been given in [14], follows here in an almost trivial way.

\subsection{Single pair production probability}

In order to derive the production probability for a single electron-positron pair with wave functions $\Phi_{e}$ and $\Phi_{p}$, we have to compute first the S-matrix element

$$
\begin{gathered}
M_{e p}=\left(b\left(\Phi_{e}\right)^{+} d\left(\Phi_{p}\right)^{+} \Omega, \mathrm{S} \Omega\right) \\
=C\left(\Omega, d\left(\Phi_{p}\right)^{+} b\left(\Phi_{e}\right)^{+} e^{S_{+-} S_{--}^{-1} b^{+} d^{+}} \Omega\right)
\end{gathered}
$$

The definition (22) and the anticommutation rules (7) enable us after some algebra to derive the commutation rules 18

$$
\begin{gathered}
b(f) e^{S_{+-} S_{--}^{-1} b^{+} d^{+}} \\
=e^{S_{+-} S_{--}^{-1} b^{+} d^{+}}\left(b(f)+d\left(\left(S_{+-} S_{--}^{-1}\right)^{+} P_{+}^{0} f\right)^{+}\right) \\
d(f) e^{S_{+-} S_{--}^{-1} b^{+} d^{+}} \\
=e^{S_{+-} S_{--}^{-1} b^{+} d^{+}}\left(d(f)-b\left(S_{+-} S_{--}^{-1} P_{-}^{0} f\right)^{+}\right),
\end{gathered}
$$

which reduce the matrix element to the form

$$
\begin{gathered}
M_{e p}=C\left(\Omega, d\left(\Phi_{p}\right) d\left(\left(S_{+-} S_{--}^{-1}\right)^{+} P_{+}^{0} \Phi_{e}\right)^{+} \Omega\right) \\
=C\left(\Phi_{e}, S_{+-} S_{--}^{-1} \Phi_{p}\right) .
\end{gathered}
$$

The matrix $\mathcal{M}=S_{+-} S_{--}^{-1}$ contains the additional scattering factor $S_{--}^{-1}$, which is missing in the literature when differential cross sections for pair production are considered. As will be explained below, $\mathcal{M}$ is identical to the result using the usual Feynman propagators.

Unfortunately, the calculation of an integral operator like $S_{--}^{-1}$ is not a trivial task, but from Eq. (15) we can derive the unitarity condition

$$
S_{-+} S_{+-}^{+}+S_{--} S_{--}^{+}=P_{-}^{0},
$$

which can be multiplied from the left by $S_{+-} S_{--}^{-1}$ leading to the equation

$$
\mathcal{M}=S_{+-} S_{--}^{+}+\mathcal{M} S_{-+} S_{+-}^{+},
$$

which can be iterated.

\section{4 n-pair production}

The vacuum-vacuum transition probability $|C|^{2}$ can be derived from

$$
1=(\mathrm{S} \Omega, \mathrm{S} \Omega)=|C|^{2}\left(e^{\mathcal{M} b^{+} d^{+}} \Omega, e^{\mathcal{M} b^{+} d^{+}} \Omega\right),
$$

with the result 17

$$
|C|^{-2}=\operatorname{det}\left(1+\mathcal{M}^{+} \mathcal{M}\right) .
$$

It is clear that the probability $P_{\text {tot }}$ to produce an arbitrary number of pairs is equal to $1-|C|^{2}$. The well-known identity

$$
\log \operatorname{det}\left(1+\mathcal{M}^{+} \mathcal{M}\right)=\operatorname{Tr} \log \left(1+\mathcal{M}^{+} \mathcal{M}\right)
$$

leads to

$$
\frac{1}{|C|^{2}}=\exp \left(-\operatorname{Tr} \sum_{n=1}^{\infty} \frac{\left(-\mathcal{M}^{+} \mathcal{M}\right)^{n}}{n}\right)
$$

or

$\frac{1}{|C|^{2}} \sum_{n=1}^{\infty} P_{n}=\frac{1}{|C|^{2}}-1=\exp \left(-\operatorname{Tr} \sum_{n=1}^{\infty} \frac{\left(-\mathcal{M}^{+} \mathcal{M}\right)^{n}}{n}\right)-1$.

Utilizing the expansion in (39) allows to write down the npair production probability by collecting all terms in (39) containing n $\mathcal{M}^{+} \mathcal{M}$ terms, e.g.

$$
P_{2}=\frac{|C|^{2}}{2}\left[\left(\operatorname{Tr}\left(\mathcal{M}^{+} \mathcal{M}\right)\right)^{2}-\operatorname{Tr}\left(\left(\mathcal{M}^{+} \mathcal{M}\right)^{2}\right)\right] \text {. }
$$

This can be understood if one takes a look at the actual calculation of the n-pair production probability analogous to the single pair production calculation presented in sect. 2.3. It follows also from the expression of the determinant in terms of antisymmetric tensor products which can be found in 19]

$$
\operatorname{det}(1+A)=\sum_{n=0}^{\infty} \operatorname{Tr}\left(\Lambda^{n}(A)\right)
$$


if $A$ is a trace-class operator. $A^{n}(A)$ is the tensor product of $A$ over the antisymmetric n-particle space.

If 'exchange terms' of the form $\operatorname{Tr}\left(\left(\mathcal{M}^{+} \mathcal{M}\right)^{n}\right)$ are neglected in (39), then the probability of producing $n$ pairs goes over into a Poisson distribution

$$
P_{n} \rightarrow e^{-\operatorname{Tr}\left(\mathcal{M}^{+} \mathcal{M}\right)} \frac{\left(\operatorname{Tr}\left(\mathcal{M}^{+} \mathcal{M}\right)\right)^{n}}{n !}
$$

which seems to be a reasonable approximation due to the correlation between the momenta of electron and positron in this case, as was also found in the calculations performed in [20]. The poisson distribution had already been derived theoretically in an earlier work 55. There, the sudden (or Glauber) approximation and a quasiboson approximation for $e^{+} e^{-}$pairs was assumed, leading to a simple but very instructive example which elucidates the pair production mechanism. It is remarkable that the Poisson distribution gives the multiplicity of particles to be

$$
N_{\text {Poisson }}=\operatorname{Tr}\left(\mathcal{M}^{+} \mathcal{M}\right)
$$

quite similar to the exact one of Eq. (28).

\subsection{Perturbation theory}

We briefly mention here the perturbative expansion of the previously discussed matrix elements. As it follows from the solution of the Dirac equation the perturbative expansion of $S_{ \pm \pm}$is given by retarded propagators

$$
\begin{gathered}
S^{r e t}(p)=\frac{\not p+m}{p^{2}-m^{2}+i p_{0} 0} \\
S_{ \pm \pm}^{(n)}(\mathbf{p}, \mathbf{q})=-i \frac{e^{n}}{(2 \pi)^{2 n-1}} P_{ \pm}^{0}(\mathbf{p}) \gamma^{0} \int d^{4} p_{1} \ldots d^{4} p_{n-1} \\
\hat{A}\left(p-p_{1}\right) S^{r e t}\left(p_{1}\right) \hat{A}\left(p_{1}-p_{2}\right) \ldots S^{r e t}\left(p_{n-1}\right) \hat{A}\left(p_{n-1}-q\right) P_{ \pm}^{0}(\mathbf{q}) .
\end{gathered}
$$

The projection operators are given in momentum space by

$$
P_{ \pm}^{0}(\mathbf{p})=\frac{1}{2 E}(E \pm \alpha \mathbf{p}+\beta m)
$$

where $\alpha$ and $\beta$ are standard Dirac matrices. The pair production amplitude, on the contrary, has to be calculated using Feynman propagators

$$
\begin{gathered}
S^{F}(p)=\frac{\not p+m}{p^{2}-m^{2}+i 0} \\
\left(S_{+-} S_{--}^{-1}\right)^{(n)}(\mathbf{p}, \mathbf{q}) \\
=-i \frac{e^{n}}{(2 \pi)^{2 n-1}} P_{+}^{0}(\mathbf{p}) \gamma^{0} \int d^{4} p_{1} \ldots d^{4} p_{n-1}
\end{gathered}
$$

$\hat{A}\left(p-p_{1}\right) S^{F}\left(p_{1}\right) \hat{A}\left(p_{1}-p_{2}\right) \ldots S^{F}\left(p_{n-1}\right) \hat{A}\left(p_{n-1}-q\right) P_{-}^{0}(\mathbf{q})$.

Eqs. (45,48) represent well-known results from (quantum) field theory which are discussed in many standard textbooks 21,22]. An explicit calculation which shows how the transition from retarded to Feynman propagators takes place when $S_{ \pm \pm}^{(n)}$ and $\left(S_{+-} S_{--}^{-1}\right)^{(n)}$ are compared can be found in [18]. It is the inverse of $S_{--}$which is responsible for this transition.

Since it is possible to solve the Dirac equation exactly in the ultrarelativistic limit, it is clear that the retarded propagator is also known in this case, and is therefore a much simpler object than the Feynman propagator from the calculational point of view.

\section{Coulomb corrections and multiplicity}

It was argued in 14 that the absence of Coulomb corrections in the high energy limit as derived in [1] could be attributed to the difference in the interpretation between the multiplicity $N=\operatorname{Tr}\left(S_{+-} S_{-+}^{+}\right)$and the single pair production probability $P_{1}=|C|^{2} \operatorname{Tr}\left(\mathcal{M}^{+} \mathcal{M}\right)$. Here we want to present some arguments that show that such an explanation cannot hold. Coulomb corrections and 'unitarity corrections' (following the terminology used in 15) can be treated as two different phenomena, which stem from different sources. Whereas unitarity corrections are due to many-particle aspects, Coulomb corrections affect the matrix element $\mathcal{M}$ directly.

Since the theory of pair production developed above is valid quite generally, we can consider the special case with two ions that have charge numbers $Z_{1}$ and $Z_{2}$, in the limit where $Z_{1} \alpha$ remains fixed but $Z_{2} \alpha$ goes to zero. Expanding in powers of $Z_{2} \alpha$ and realizing that the potential of the ion with charge $Z_{1} e$ is a static Coulomb potential in its rest frame, we find that both $S_{-+}$and $S_{+-}$are at least of order $Z_{2} \alpha$. Using the recursion relation of Eq. (34), the second term on the right hand side is at least of order $\left(Z_{2} \alpha\right)^{3}$ and can therefore be neglected. Therefore, if only contributions at first order in $Z_{2} \alpha$ are kept (whereas contributions at higher orders in $Z_{1} \alpha$ are important, as it is illustrated in Fig. 2), the matrix element for pair production is found to be $\mathcal{M} \approx S_{+-} S_{--}^{+}$. This can also be seen directly from the definition $\mathcal{M}=S_{+-} S_{--}^{-1}$ and the fact that for a static field we have no off-diagonal elements in $S$ with respect to the block structure given in Eq. (15) and therefore $S_{--}^{-1}=$ $S_{--}^{+}$. Summing over all final states we get for $P_{1}$ (keeping in mind that at this order of $Z_{2} \alpha$ also $|C|^{2}=1$ )

$$
P_{1} \approx \operatorname{Tr}\left(S_{+-} S_{--}^{+} S_{--} S_{-+}^{+}\right)=\operatorname{Tr}\left(S_{+-} S_{-+}^{+}\right) .
$$

Following the arguments for the high energy limit of [1] this probability should now be identical to the lowest order result. On the other hand the limit performed here corresponds exactly to the one which is done in order to derive the Bethe-Maximon theory 23, 10]. Therefore the discrepancy between the two results is also present in this case, independently of the many-particle aspects. Comparing the two multiplicities $N$ and $N_{\text {Poisson }}$ one finds that they start to disagree only at fourth order in $Z_{2} \alpha$.

Therefore one has to conclude that the discrepancies in the literature concerning Coulomb corrections cannot be traced back to the many-body aspects of pair production in this case. 

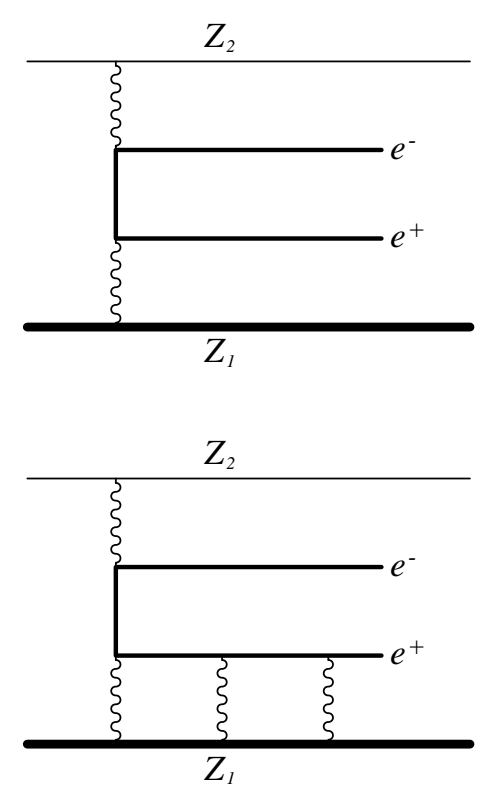

Fig. 2. Examples of graphs contributing to pair production for $Z_{2} \alpha \ll 1$.

\section{Conclusion and Outlook}

In this paper we have derived in a very straightforward way the link between the single-particle $S$ matrix elements obtained from the solution of the Dirac equation and its relation to the multiplicity and single-pair production probability. The term $S_{--}^{-1}$, which is missing in many papers and which is important for the calculation of the differential cross section with respect to both electrons and positrons, was introduced. A careful analysis of the limit which is relevant for a comparison with the BetheMaximon results shows that the absence of Coulomb corrections in this high energy limit cannot be understood by a purely conceptional treatment of the external field problem. The problem therefore seems to lie in the way this high energy limit is made. That a too simplified use of the eikonal approximation leads to a result in contradiction with the Bethe-Maximon one was already pointed out in [24], whereas within a more refined analysis as given in 25 there is no disagreement.

In priniciple the pair production in external fields is well understood, but the numerical evaluation of the Coulomb corrections for two highly charged ions, especially at small impact parameter, still needs to be found.

\section{References}

1. B. Segev, J.C. Wells, Phys. Rev. C 59, no. 5, 2753 (1999)

2. A. J. Baltz, L. McLerran, Phys. Rev. C 58, 1679 (1998)

3. R. P. Feynman, Phys. Rev. 76, 749 (1949)

4. J. Schwinger, Phys. Rev. 93, 615 (1954)

5. G. Baur, Phys. Rev. A 42, no. 9, 5736 (1990)

6. K. Hencken, D. Trautmann, G. Baur, Phys. Rev. C 59, 841 (1999)
7. M. J. Rhoades-Brown, J. Weneser, Phys. Rev. A 44, 330 (1991)

8. C. Best, W. Greiner, G. Soff, Phys. Rev. A 46, 261 (1992)

9. K. Hencken, D. Trautmann, G. Baur, Phys. Rev. A 51, 998 (1995)

10. D.Yu. Ivanov, K. Melnikov, Phys. Rev. D 57, 4025 (1998)

11. D.Yu. Ivanov, A. Schiller, V.G. Serbo, Phys. Lett. B 454, 155 (1999)

12. U. Eichmann, J. Reinhardt, W. Greiner, Phys. Rev. A 61, 062710 (2000)

13. R.N. Lee, A.I. Milstein, Phys. Rev. A 61, 032103 (2000)

14. A.J. Baltz, F. Gelis, L. McLerran, A. Peshier, Nucl. Phys. A 695, 395 (2001)

15. R.N. Lee, A.I. Milstein, V.G. Serbo, hep-ph/0108014

16. A.J. Baltz, Phys. Rev. Lett. 78, no. 7, 1231 (1997)

17. H. P. Seipp, Helvetica Physica Acta 55, 1 (1982)

18. G. Scharf, Finite quantum electrodynamics: the causal approach (Springer, New York, 1995) (second edition)

19. M. Reed, B. Simon, Methods of Modern Mathematical Physics Vol. IV: Analysis of Operators (Academic Press, New York, 1978), p. 323, eq. (188)

20. K. Hencken, D. Trautmann, G. Baur, Phys. Rev. A 51, 998 (1995)

21. C. Itzykson, J.-B. Zuber, Quantum Field Theory (McGraw-Hill, 1980), p. 89

22. N.N. Bogoliubov, D.V. Shirkov, Introduction to the Theory of Quantized Fields (Interscience Publishers, Inc., New York, 1959)

23. H.A. Bethe, L. Maximon, Phys. Rev. 93, 768 (1954)

24. R. Blankenbecler, S. D. Drell, Phys. Rev. D 36, 2846 (1985)

25. B.Z. Kopeliovich, A.V. Tarasov, O.O. Voskresenskaya, Eur. Phys. J. A 11, 345 (2001) 